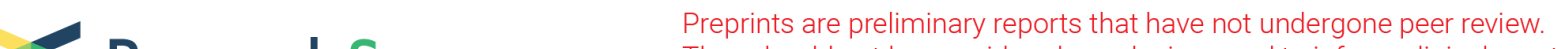 $\begin{array}{ll}\text { Research Square } & \text { They should not be considered conclusive, used to inform clinical practice, } \\ \text { or referenced by the media as validated information. }\end{array}$
}

\section{The Prevalence of Human Immunodeficiency Virus Infection among Voluntary Blood Donors in Mainland China: A Systematic Review and Meta- Analysis}

\section{Zhan Gao}

Institute of Blood Transfusion Chinese Academy of Medical Sciences Peking Union Medical College: Chinese Academy of Medical Sciences \& Peking Union Medical College Institute of Blood Transfusion Yuhui Li

Institute of Xi'an Blood Bank

\section{Liu Zhiyang}

Institute of Blood Transfusion Chinese Academy of Medical Sciences Peking Union Medical College: Chinese Academy of Medical Sciences \& Peking Union Medical College Institute of Blood Transfusion

\section{Yang Huang}

Institute of Blood Transfusion Chinese Academy of Medical Sciences Peking Union Medical College: Chinese Academy of Medical Sciences \& Peking Union Medical College Institute of Blood Transfusion Miao He ( $\nabla$ hemiao@ibt.pumc.edu.cn )

Institute of Blood Transfusion Chinese Academy of Medical Sciences Peking Union Medical College: Chinese Academy of Medical Sciences \& Peking Union Medical College Institute of Blood Transfusion

\section{Research}

Keywords: HIV/AIDS, prevalence, China

Posted Date: November 1st, 2021

DOl: https://doi.org/10.21203/rs.3.rs-903053/v1

License: (1) This work is licensed under a Creative Commons Attribution 4.0 International License. Read Full License 


\section{Abstract}

BACKGROUND: The epidemic of Human Immunodeficiency Virus (HIV) in China has been increasing. Despite the implementation of screening strategies, there was still the residual risk of transfusiontransmitted HIV. This study investigated the HIV prevalence among voluntary blood donors during the past 24 years in order to characterize the epidemiology of HIV.

METHODS: The literature concerning the HIV screening reactive rate and prevalence in Chinese voluntary blood donors were collected through the systematic searching of four electronic databases. After integrating data, following the Preferred Reporting of Items for Systematic Reviews and Meta-Analyses guidelines, data manipulation and statistical analyses were conducted by Stata 12.0. We investigated the trend of HIV prevalence (and 95\% Cls) and related epidemiological characteristics among blood donors in mainland China.

RESULTS: The results indicated that HIV prevalence was $0.0165 \%(95 \% \mathrm{Cl}, 0.0155 \%-0.0176 \%)$ with a remarkable rise, which varied from $2000(0.0034 \%)$ to 2017 (0.0247\%). Importantly, the heterogeneity was found in occupations and Donation frequency. Additionally, the HIV prevalence were significantly higher among male donors than among female donors.

CONCLUSIONS: Our results indicated obvious difference between HIV screening reactive rate and HIV prevalence in Chinese blood donors and HIV screening reactive rate cannot reflect reality of HIV infection. In this study, continuous increased trend of HIV prevalence demonstrates extended residual risk of blood transfusion, and the whole society is supposed to pay close attention to HIV infection.

\section{Background}

It is known to all that Acquired Immune Deficiency Syndrome (AIDS) is one of the most severe pandemics. It was estimated that approximately 37.9 million people were infected with human immunodeficiency virus (HIV) and 1.7 million annually were new infections. ${ }^{1}$ In China, there were sixtyfour thousand people infected with HIV or diagnosed with AIDS in 2018, eighteen thousand patients died of AIDS. ${ }^{2}$ Since the Peking Union Medical College Hospital reported the first case of AIDS in China in 1985 , The infection of HIV has spread all over the country, ${ }^{3}$

In 1988, Notice on Rectifying the Production Management of Blood Products promulgated by the Ministry of Health required testing of blood donors for HIV antibodies. After the implementation of the blood donation law in China in 1998, the government advocated voluntary blood donation and banned paid blood donations for clinical use, these Policies undoubtedly reduces the risk of HIV transmission through blood transfusion.

Meanwhile, the advances in pathogen detection technology have decreased risks of transfusiontransmitted infections (TTIS), which enhanced blood safety. ${ }^{4-7}$ The routine blood screening included two rounds of parallel post-donation enzyme-linked immunosorbent assay (ELISA) from different 
manufacturer. If test results in anti-HIV is positive for one or two kits, the reexamination will be conducted. When the result is still positive, the sample will be determined as screening positive, and then will be sent to the City Center for Disease Control and Prevention (CDC) for anti-HIV confirmative test using western blot (WB).

Besides the serological testing (ELISA), the of Nucleic acid testing (NAT) was introduced into the postdonation screening in 2010 in China. NAT could shorten the window period and reduce the transfusion residual risks. With all these efforts, transfusion-transmitted risk of HIV infection has been reduced compared to the past. However, there are a considerable amount of HIV infection cases in China, and HIV infection through transfusion remains an imperative challenge for blood safety in China. A wide range of studies aimed at understanding the epidemiology and related risk factors of HIV among voluntary blood donors in China have been published, but some defects were found in these studies, such as contradictory results from difference studies and deficient representative samples. Additionally, due to insufficient sample size or invalid statistical methods, some studies were unqualified. Thus, it is essential to examine the epidemiology of HIV among the blood donors in a systematic approach. Meta-analysis is chosen as the most reasonable means to address this issue, because Meta-analysis is a statistical method which is used to combine findings from plentiful independent studies on HIV infection among blood voluntary donors in multiple regions.

Through meta-analysis, we have investigated the HIV blood screening reactive rate and prevalence, and obtained the demographics of blood voluntary donors in China. This study is regard to a nationwide surveillance of AIDS cases and HIV infections among Chinese voluntary blood donors with revealing of heterogeneity between previous studies during the past 24 years in mainland China.

\section{Materials And Methods}

Literature selection and data extraction

The inclusion criteria were as follows: 1) The study was conducted only in mainland China (Hong Kong, Taiwan, and Macao were excluded); 2) The HIV screening test should is ELISA or NAT and screening strategy is supposed to do conform with relevant standardized donor screening policies decreed by government; 3) Studies included HIV screening reactive rate or prevalence with the WB confirmatory method; and 4)Experimental objects are blood voluntary donors.

We excluded studies if they were overlapping or paradoxical (i. e. data from literature were repeat or inconsistent between the context); they lacked exact sample size, specific research time or location; the sample size was less than 50 individuals; their objects consisted of plasma donors for manufacturers; or if the unit of measure was $U$ (related to blood collection). We also excluded studies if their population was not entirely wholly blood voluntary donors.

All titles and abstracts identified in the search were independently examined by two experienced investigators. The same two investigators then assessed the full text of any articles deemed potentially 
relevant, and then the disagreements between investigators were resolved by consulting a third investigator to reach a consensus.

\section{Literature search}

We commenced designing and conducting this meta-analysis based on the proposed PRISMA guidelines. The search of electronic literatures on HIV epidemiology among blood voluntary donors in mainland China was performed using China National Knowledge Infrastructure, Medline, PubMed, and Wanfang Data from 1988 to 2018 with key words including "HIV", "human immunodeficiency virus" or "AIDS"; "blood"; "blood donors"; "blood centers"; or "donation"; "transfusion transmitted disease"; "screening reactive" or "disqualification"; "epidemiology"; and "China" or "Mainland China". No language restrictions were applied.

Quality assessment of the studies

In consideration of the applicability of quality assessment, all included studies were assessed for methodologic quality using the prevalence critical appraisal instrument developed by Munn and colleagues. ${ }^{8}$

\section{Statistical analysis}

Pooled results for HIV prevalence and corresponding 95\% confidence were used to assess the condition of HIV infection in mainland China. The heterogeneity among studies was tested by the $\mathrm{I}^{2}$ test and Q statistical test. On account of the high heterogeneity of collected studies in our research $\left(1^{2}>90 \%, p<\right.$ $0.1)$, DerSimonian-Laird random-effects model was used to calculate the pooled odds ratios. Metaregression analysis based on multiple factors (publication year, province, sex ratio, sample size) was performed to find the source of heterogeneity. In addition, publication bias was assessed by Egger's tests, in which $p<0.05$ represents significance.

Characteristic-based stratified analysis was performed and the $Z$ or chi-square test was used to calculate statistical difference among subgroups. All data analysis were performed with computer software (Stata Statistical Software Package 12.0, StataCorp) for the statistical analysis. The PRISMA flow diagram and geographical distribution maps were made by Adobe Photoshop 12.0 (http:// www.adobe.com/), and the trend plot was made by Joinpoint Regression Program (https://surveillance.cancer.gov/help/joinpoint).

\section{Results}

\section{The selection of literatures}

According to the inclusion and exclusion criteria, 394 studies of individual voluntary blood donors were identified in meta-analysis. We collected demographic characteristics from these studies including district, sexuality, age, ethnicity, vocation, educational level, marriage, and blood type. Additional 
information including title, author, study year, sample capacity, the quantity of subjects with people Living with HIV(PLWH) was extracted. (Fig, 1)

\section{General Information}

Our study included approximately 1.1 hundred million voluntary blood donors, demographic data from donors was tabulated, as presented in Table 1. 
Table 1

Demographic characteristics of voluntary blood donors

\begin{tabular}{|ll|}
\hline Donation characteristics & Percentage \\
\hline Male & \\
\hline Female & 61.88 \\
\hline Age & 38.12 \\
\hline 18-30 & \\
\hline $31-40$ & 51.87 \\
\hline$>41$ & 27.02 \\
\hline Ethnicity & 21.10 \\
\hline Han & \\
\hline Minorities & 94.34 \\
\hline Blood group & 5.66 \\
\hline A & \\
\hline B & 28.60 \\
\hline AB & 28.28 \\
\hline O & 8.98 \\
\hline Education & 34.15 \\
\hline Junior college, Bachelor degree or above & 41.20 \\
\hline Senior Middle School & 31.28 \\
\hline Junior Middle School and below & 27.53 \\
\hline Occupation & \\
\hline Student & 13.94 .96 \\
\hline Soldier & 1.74 \\
\hline Farmer & \\
\hline Business Service Personnel & \\
\hline Worker & \\
\hline
\end{tabular}




\begin{tabular}{|ll|}
\hline Donation characteristics & Percentage \\
\hline Freelancer & 13.95 \\
\hline Unemployed & 0.66 \\
\hline Donation frequency & \\
\hline First-time donor & 53.04 \\
Repeat donor & 46.96 \\
Marriage & \\
\hline Married person & 53.24 \\
Single & 46.76 \\
\hline
\end{tabular}

In this study, all the 31 provinces, autonomous regions and municipalities in mainland China were divided into seven areas: East China, North China, South China, Central China, Northwest China, Southwest China, and Northeast China.

\section{Hiv Prevalence}

After screening, all the HIV-associated cases were confirmed by Western Blot (WB). 394 studies were included into the investigation, the pooled statistical result of HIV prevalence is presented in Table 2. 
Table 2

Pooled HIV prevalence in voluntary blood donors in different aeras of Chinese mainland

\section{HIV prevalence}

\begin{tabular}{|c|c|c|c|}
\hline Study location & province & Number in study (number of donors) & HIV prevalence ${ }^{A}$ \\
\hline \multirow[t]{7}{*}{ East China } & Shandong & $35(6316988)$ & $0.0074(0.0055-0.0092)$ \\
\hline & Anhui & $10(1501127)$ & $0.0095(0.0052-0.0137)$ \\
\hline & Jiangsu & $25(5418925)$ & $0.0101(0.0079-0.0123)$ \\
\hline & Zhejiang & $32(7094282)$ & $0.0115(0.0091-0.0139)$ \\
\hline & Fujian & 13(1691856) & $0.0130(0.0072-0.0188)$ \\
\hline & Jiangxi & 13(1620195) & $0.0099(0.0067-0.0132)$ \\
\hline & Shanghai & $1(16282)$ & $0.0061(-0.0059-0.0182)$ \\
\hline \multirow[t]{3}{*}{ South China } & Guangdong & $40(11439854)$ & $0.0160(0.0127-0.0193)$ \\
\hline & Guangxi & $22(5820684)$ & $0.0382(0.0312-0.0452)$ \\
\hline & Hainan & $2(947447)$ & $0.0118(0.0072-0.0164)$ \\
\hline \multirow[t]{5}{*}{ North China } & Hebei & $12(4388678)$ & $0.0075(0.0048-0.0101)$ \\
\hline & Beijing & $5(1049231)$ & $0.0405(0.0240-0.0569)$ \\
\hline & Shanxi & 11(2310420) & $0.0206(0.0117-0.0295)$ \\
\hline & Tianjin & $8(2366138)$ & $0.0241(0.0152-0.0329)$ \\
\hline & Inner Mongolia & $13(364117)$ & $0.0115(0.0044-0.0185)$ \\
\hline \multirow[t]{3}{*}{ Central China } & Hunan & 11(2079521) & $0.0165(0.0111-0.0220)$ \\
\hline & Hubei & 10(1791538) & $0.0097(0.0074-0.0119)$ \\
\hline & Henan & 25(5701731) & $0.0150(0.0126-0.0175)$ \\
\hline \multirow[t]{5}{*}{ Southwest } & Sichuan & 17(2191210) & $0.0318(0.0226-0.0410)$ \\
\hline & Yunnan & $12(631797)$ & $0.0743(0.0507-0.0979)$ \\
\hline & Tibet & $3(65476)$ & $0.0340(0.0198-0.0481)$ \\
\hline & Guizhou & $9(846424)$ & $0.0342(0.0211-0.0473)$ \\
\hline & Chongqing & $10(635136)$ & $0.0146(0.0070-0.0222)$ \\
\hline \multirow[t]{2}{*}{ Northwest } & Shaanxi & 14(3514629) & $0.0152(0.0100-0.0205)$ \\
\hline & Gansu & 11(1320309) & $0.0122(0.0075-0.0170)$ \\
\hline
\end{tabular}




\begin{tabular}{|llll|}
\hline \multicolumn{3}{|c|}{ HIV prevalence } \\
\hline & Ningxia & $6(302650)$ & $0.0064(-0.0022-0.0151)$ \\
\hline & Xinjiang & $7(458247)$ & $0.0255(0.0163-0.0346)$ \\
\hline & Qinghai & $4(371624)$ & $0.0253(0.0140-0.0366)$ \\
\hline & Heilongjiang & $1(14097)$ & $0.0071(-0.0068-0.0210)$ \\
\hline Pooled & Liaoning & $11(36179850)$ & $0.0115(0.0063-0.0167)$ \\
\hline A Data are reported as percent(95\%Cl) & $1(75404)$ & $0.0027(-0.0010-0.0063)$ \\
\hline
\end{tabular}

The overall pooled prevalence among voluntary blood donors in mainland China was $0.0165 \%(95 \% \mathrm{Cl}$, $0.0155 \%-0.0176 \%$ ) with a broad range of $0.0027-0.0743 \%$ in different regions. There was an evident regional difference in HIV prevalence among blood voluntary donors, in which Southwest China $(0.0390 \%$, 0.0325\%-0.0456\%) and South China(0.0219\%, 0.0187\%-0.0251\%)had a relatively higher HIV prevalence, while the lowest HIV prevalence was shown in East China(0.0098\%, 0.0087\%-0.0109\%). Moreover, Figures 2 indicated geographical distribution of HIV prevalence, and the overall HIV prevalence varied annually, which was shown. (Fig. 3)

\section{Demographics Of Hiv Infection Confirmed Voluntary Donors}

Age

A total of 21 studies about age were included in the examination of the association between HIV prevalence and age among voluntary blood donors. Respectively, the prevalence of donors aged 18-30,3140, over 41 were $0.0131 \%$ (95\% $\mathrm{Cl}, 0.0098 \%-0.0165 \%), 0.0171 \%(95 \% \mathrm{Cl}, 0.0118 \%-0.0223 \%)$ and $0.0078 \%$ $(95 \% \mathrm{Cl}, 0.0046 \%-0.0109 \%)$.There was no marked difference among voluntary groups aged $18-30 \bowtie 31-40$, over 41. $(p>0.05)$.

Gender

There were 64 studies brought into the analysis of the correlation between HIV prevalence and sex among voluntary blood donors. The prevalence in male voluntary donors was $0.0224 \%(95 \% \mathrm{Cl}$, $0.0194 \%-0.0254 \%)$, and that in female donors was $0.0057 \%(95 \% \mathrm{Cl}, 0.0045 \%-0.007 \%)$. Conspicuous discrepancy was observed between the gender $(p<0.05)$.

Ethnicity 
We investigated the association of HIV prevalence and ethnicity, and acquired HIV prevalence and $95 \% \mathrm{Cl}$ in the blood voluntary groups of which Han $0.0206 \%(0.0118 \%-0.0294 \%)$ and ethnic minority $0.0403 \%$ $(0.0215 \%-0.0591 \%)$. Nevertheless, no obvious differences were found in Han and ethnic minority donors $(p>0.05)$.

Marriage

According to examination, HIV prevalence in married and unwed donors were $0.0213 \%(95 \% \mathrm{Cl}$, $0.0150 \%-0.0275 \%), 0.0191 \%(95 \% \mathrm{Cl}, 0.0115 \%-0.0267 \%)$. there was no significant difference between married and unwed donors $(p>0.05)$.

Occupation

A total of 22 studies were involved in the analysis of correlation between HIV prevalence and donors' occupation. The business service personnel had the highest HIV prevalence $(0.0287 \% ; 95 \% \mathrm{Cl}$, $0.0107 \%-0.0467 \%)$, while the lowest $(0.0075 \% ; 95 \% \mathrm{Cl}, 0.0055 \%-0.0095 \%)$ were students. The HIV prevalence of civil servants, farmers, freelancers, and workers were $0.0090 \%(95 \% \mathrm{Cl}, 0.0058 \%-0.0122 \%)$, $0.0175 \%(95 \% \mathrm{Cl}, 0.0089 \%-0.0262 \%), 0.0127 \%$ (95\% Cl, 0-0.0256\%), $0.0103 \%$ (95\% Cl, $0.0054 \%-0.0151 \%)$. It was important that significant differences $(p<0.05)$ were observed between business service personnel and the other vocational groups.

\section{Education}

The analysis about relevance of HIV prevalence and the education was conducted in accordance with 20 related studies about education. The individuals of Junior Middle School and below had the highest HIV prevalence $(0.0201 \% ; 95 \% \mathrm{Cl}, 0.0146 \%-0.0257 \%)$, compared with the group of Junior college, Bachelor degree or above $(0.0121 \% ; 95 \% \mathrm{Cl}, 0.0085 \%-0.0157 \%)$ and Senior Middle School $(0.0133 \% ; 95 \% \mathrm{Cl}$, $0.0092 \%-0.0174 \%)$. No significant difference was found in different groups of education level $(p>0.05)$.

Donation frequency

We identified 22 studies associated with HIV prevalence and donation frequency. Through statistical treatment, the HIV prevalence of first donation $(0.0219 \% ; 95 \% \mathrm{Cl}, 0.0172 \%-0.0267 \%)$ higher than the repeat donation $(0.0087 \% ; 95 \% \mathrm{Cl}, 0.0062 \%-0.0112 \%)$. Besides, there was a Statistic difference between first and repeat donors $(p<0.05)$.

\section{Year}

The overall HIV prevalence showed a increasing trend from 1998 to 2017. As shown in Fig. 3, the Joinpoint trend for HIV prevalence decreased noticeably, by $-63.9 \%(95 \% \mathrm{Cl},-89$ to 18.2$)$ per year from before 1998 through 2000; ascended reversely, by $27.1 \%(95 \% \mathrm{Cl},-43.6$ to 186.2) annually during 2001 through 2003; later increased in small increments, by $4.1 \%(95 \% \mathrm{Cl},-10.8$ to 21.5$)$ per year during 2004 through 2008; and then augmented by $12.2 \%(95 \% \mathrm{Cl}, 9$ to 15.6) per year during 2009 through 2017. 
A wide range of heterogeneity $\left(I^{2}>90 \%, p<0.1\right)$ existed in included studies about HIV prevalence. The meta-regression analysis based on multiple factors (publication year, province, sex ratio [male/female], and sample size) showed that the variable included in the regression was province $(p<0.05)$, which probably explain the part of heterogeneity.

\section{Publication bias}

We assessed the publication bias through Egger's test, acquired the meaningful publication bias $(\mathrm{p}<$ 0.05). Subsequently, the detailed analysis of publication bias for subgroups was developed: In geographic locations, the obvious publication bias $(p<0.05)$ existed in 11 provinces; In occupation group, publication bias was found in farmer, business services and unemployed group; There was a publication bias $(p<0.05)$ in married individuals. In addition, significant publication bias $(p<0.05)$ occurred in the groups of gender, age, education and donation frequency, no significant publication bias $(p>0.05)$ was found in the rest of groups or subgroups.

Besides, we investigated statistical characteristics of HIV screening of voluntary blood donors (Table S1) and analyzed associated factors (e.g., geographical distribution and annual variation, see Supplementary Figure 1-2), See appendix for details.

\section{Discussion}

In mainland China, the provision of blood primarily depended on paid and employer-organized donations before 1998. When the "Blood Donation Law of the People's Republic of China" came into force since 1998, the government had banned all kinds of remunerative blood donation and HIV prevalence rate decreased significantly in blood donors. However, prevalence later edged up until 2017, which may attribute to an increasing amount of PLWH. According to data compiled by Chinese Center for Disease Control and Prevention (CDC) and previous reports, the top five provinces with the largest amount of HIVinfected people are Sichuan, Yunnan, Guangxi, Guangdong, and Henan, ${ }^{9-11}$ which was consistent with the highest prevalence among voluntary blood donors found in Yunnan (Southwest), Guangxi (South), Sichuan (Southwest), etc.

In our study. The detailed information about HIV prevalence can be seen in Table 2. Notably, there was significant difference between HIV screening reactive rate (see appendix) and prevalence in Qinghai, Shaanxi, Ningxia, Gansu, and Chongqing, which demonstrates that there are high false positives in HIV screening testing in these regions. The above-mentioned situation should be of concern to the community. In addition, The PLWH and the high-risk groups with high mobility threatens public health security. ${ }^{12}$ Because of Yunnan, Sichuan, Guangxi, which are characterized by high HIV prevalence are the labor exporting provinces, the government is supposed to strengthen capacity of HIV prevention and monitoring in these regions. For one thing, the deficiency of efficient prevention strategies and attention to high-risk groups might have contributed to the higher prevalence in areas with high incidence rates in 
China. ${ }^{13}$ For another, due to poor public health awareness of HIV/AIDS and limitation of medical treatment, which may result in the continuing increase of HIV/AIDS infection. ${ }^{14-16}$

In subgroup analysis, there are some marked differences in HIV prevalence among voluntary donors regarding occupation and donation frequency. HIV prevalence were lower for repeated blood donors than for first-time blood donation $(\mathrm{p}<0.05)$, and the results remain the same as in previous reports. ${ }^{17-19}$ That's because many first blood donors with certain high-risk behaviors for HIV/AIDS donated blood with the purpose of physical examination, and led to an increase in the HIV-positive rate among the first blood donors. During the study period, the amount of first blood donors was more than repeated blood donors, which also caused higher residual risk of the first blood donors than repeated blood donors. In terms of occupation, HIV prevalence in business service group is the highest and there is a statistical difference between business service and the other occupations $(p<0.05)$. Maybe the difference was as a result of different levels of knowledge about blood donation and HIV/AIDS among all walks of life, and sex workers related to business service industry are vulnerable to HIV infection. ${ }^{20}$

Moreover, there was a discrepancy between male and female donors in HIV prevalence, $(p<0.05)$ and this is most probably due to heterosexual transmission and Men who have sex with men (MSM) group. Firstly, the fast-growing share of sexual transmission is very worrying, ${ }^{21}$ and HIV infection cases by Heterosexual transmission account for about $65 \%$ of all cases in the national work report of AIDS in $2016 .^{22}$ It is worth noting that the main mode of heterosexual transmission is through commercial sexual activity. ${ }^{23}$ Secondly, HIV/AIDS epidemic among MSM has been on the rise in mainland China in recent years. ${ }^{24,25}$

We may apply the following measures to reduce HIV blood transfusion risk: ${ }^{26} 1$ ) designing a first-time donor's follow-up mechanism to inspire more first-time donors to be converted into repeat donations; 2 ) improving linked deferred donor registries that will help to defer donors who previously were identified as HIV reactive at different locations; 3 ) continuing the nationwide implementation of NAT in routine screening to detect People Living with HIV(PLWH); and 4) educating donor on the awareness of HIV prevention, such as safe sexual behaviors. These will make a valuable contribution to ensure Chinese blood safety.

In our study, it should be noted that there was a discrepancy between HIV screening reactive rate and HIV prevalence. One possible explanation for this may be linked to technology of HIV test. ELISA is used to detect blood borne viruses in blood donation service, and the judgement results is based on the ratio of signal to Cut-off(S/CO). Generally, $\mathrm{S} / \mathrm{CO} \geq 1$ is identified as positive; the $\mathrm{S} / \mathrm{CO}<1$ is reported as negative. For preventing missing detection, many institutions and laboratories lowered Cut off value to $70-90 \%$, namely setting up gray zone. ${ }^{27}$ Considering multiple factors such as restricted sensibility and low specificity of detection reagents, window period and blood donation group for test-seekers, gray zone was established to ensure blood security. ${ }^{28}$ On the one hand, for the sake of avoiding omission of false negative samples, the setting up of gray zone is beneficial to efficiently detect suspicious samples, reduce 
risk of missing detection and secure blood safety. ${ }^{29,30}$ On the other hand, the establishment of gray zone provoked an increasing of screening rate of false positives in ELISA, obtained information which is inconsistent with the fact of HIV screening and created wasted resources. ${ }^{31}$ In addition, the uniform standard for setting of gray zones has not yet been fully developed in China. Although the establishment of gray zone has its limitations, it is of tremendous importance in enhancing blood safety in China in view of economic cost and social influence. Fortunately, with the introduction of NAT and more sensitive and more specific ELISA assays, the rate of screening reactive has decreased.

Most importantly, the findings indicated that HIV prevalence in voluntary blood donors has continued to rise over time, and was consistent with the data from Chinese annual national notifiable infectious disease report. The situation of HIV infection of voluntary blood donors could indirectly reflect that of general population. Consequently, the issue about HIV infection in general population remains serious, and addressing residual risk of blood transfusion still be a great challenge. The problem of HIV infection must not be overlooked, which requires continuous joint efforts of government, institutions, and society to reduce the threat to public health. On a positive note, in view of such a grave situation of HIV infection, the number of cases of transfusion transmissible infections has dropped drastically thanks to a new generation of ELISA reagents and NAT.

Overall, the heterogeneity cannot be eradicated or avoided in meta-analysis, particularly those from crosssectional studies with wide distribution and long-term period. ${ }^{32}$ In regard to heterogeneity in this study, random-effects models was used to eliminate the effect of heterogeneity as much as possible. Additionally, we further analyzed the potential sources of heterogeneity through subgroup with metaregression method, and the results showed that province may contribute more heterogeneity than other groups.

In our study, all selected literatures were rigorously screened and were qualified for the meta-analysis. Publication bias were determined in some subgroups. Remarkably, there were less than five published reports in some provinces, which may weaken the effect of the meta-analysis. However, some literatures must be excluded owing to the low quality and lack of relevant statistics. In addition, this study only included literatures published in Chinese and English, which may relatively play a critical part in the publication bias. Even so, a comprehensive search strategy combined with the large sample size and various resources of literature in this study may have been conducive to minimization of publication bias.

The meta-analysis results presented here come with some inherent limitations: 1) All studies used crosssectional observational study design. 2) Most of the literatures was restricted to Chinese language and very few in English. 3) Some confusing information from low quality literatures could not be confirmed and had to be excluded for failure to contact the authors.

\section{Conclusions}


We found there were differences between HIV screening and prevalence not only in the rate but also by the time. And in recent 10 years the prevalence of HIV was rising steadily.On the basis of the results in this study, we suggest that there was a potential HIV rising in recent years in the general population which was a great threat to the public health. The strategy of blood discarding about HIV screening should be reviewed carefully which should balance the blood supply and blood safety under existing conditions.

\section{Abbreviations}

HIV: Human Immunodeficiency Virus $₫ A I D S:$ Acquired Immune Deficiency Syndrome $\ T T$ : transfusion-

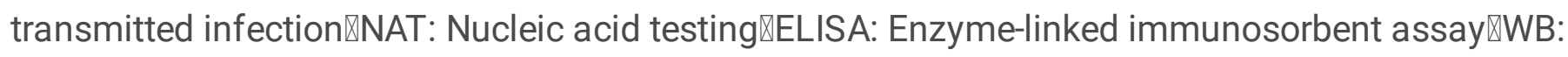

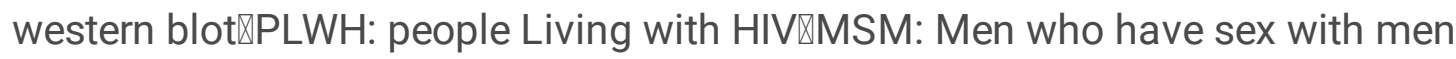

\section{Declarations}

\section{Ethics approval and consent to participate}

Not applicable.

\section{Consent for publication}

All authors read and approved the final manuscript.

\section{Availability of data and materials}

The datasets analyzed during the current study are available from the corresponding author on reasonable request.

\section{Competing interests}

The authors declare that they have no competing interests

\section{Funding}

This work was supported by Key Research and Development Projects of Science and Technology Department of Sichuan Province (No. 2019YFS0319).

\section{Authors' contributions}

ZG was responsible for designing and conducting this work, $Y L$ analyzed data and wrote this manuscript. ZL prepared data extraction. YH was involved in the investigation as a third investigator. $\mathrm{MH}$ was the principal investigator and primarily responsible for all aspects of research design and coordination and contributed to drafting the manuscript. All authors read and approved the final manuscript.

\section{Acknowledgements}




\section{References}

1. Joint United Nations Progamme on HIV/AIDS. Fact sheet: latest statistics on the status of the AIDS epidemic. http://www.unaids.org/en/resources/fact-sheet. Accessed July 31, 2019.

2. China Ministry of Public Health. Estimated report of HIV/AIDS epidemic in China 2018. http://www.moh.gov.cn. Accessed 17 Feb 2019.

3. State Council AIDS Working Committee Office and UN Theme Group on HIV/AIDS in China. A joint assessment of HIV/AIDS prevention, treatment and care in China. Beijing: China Ministry of health 2004.

4. Soldan K, Davison K, Dow B. Estimates of the frequency of HBV, HCV, and HIV infectious donations entering the blood supply in the United Kingdom, 1996 to 2003. Euro Surveill. 2005; 10: 17-19.

5. Pillonel J, Laperche S, Etablissement Français du sang. Trends in risk of transfusion-transmitted viral infections (HIV, HCV, HBV) in France between 1992 and 2003 and impact of nucleic acid testing (NAT). Euro Surveill. 2005; 10: 5-8.

6. Li W, Gao Z, Yang C, et al. The estimation of prevalence, incidence, and residual risk of transfusiontransmitted human hepatitis B infection from blood donated at the Anhui blood center, China, from 2009 to 2011. PLoS One. 2013; 8: e73472.

7. Li C, Xiao X, Yin $\mathrm{H}$, et al. Prevalence and prevalence trends of transfusion transmissible infections among blood donors at four Chinese regional blood centers between 2000 and 2010. J Transl Med. 2012; 10: 176.

8. Munn Z, Moola S, Riitano D, et al. The development of a critical appraisal tool for use in systematic reviews addressing questions of prevalence. Int J Health Policy Manag. 2014; 3: 123-128.

9. The State Council of the People's Republic of China. Notice on further strengthening the prevention and treatment of AIDS. 2010; No.48 of the State Council『Available at http://www.gov.cn/zwgk/201102/16/content_1804536.htm. Accessed Sep 16, 2020.

10. Gao Y, Feng XF, Wen J, et al. Spatio-temporal distribution and correlation of reported cases of hepatitis C and HIV/AIDS in China, 2012-2017. Chinese Journal of Epidemiology. 2019; 40: 155-159.

11. Wang LY, Qin QQ, Ding ZW, et al. Current Situation of AIDS epidemic in China. Chin J AIDS STD. 2017; 23: 330-333.

12. Lv F, Fu J, Zhang R, et al. Analysis of HIV epidemic situation and immigrant population. Infect Dis Info. 2006; 19: 259-261.

13. Wang N. HIV Epidemic in China and the world: Current situation and challenges. Science \& Technology Review. 2005; 23: 4-8.

14. Schranz AJ, Barrett J, Hurt CB, et al. Challenges Facing a Rural Opioid Epidemic: Treatment and Prevention of HIV and Hepatitis C. Curr HIV/AIDS Rep. 2018; 15: 245-254. 
15. Peteet B, Staton M, Miller-Roenigk B, et al. Rural Incarcerated Women: HIV/HCV Knowledge and Correlates of Risky Behavior. Health Educ Behav. 2018; 45: 977-986.

16. Henderson ER, Subramaniam DS, Chen J. Rural-Urban Differences in Human Immunodeficiency Virus Testing Among US Adults: Findings from the Behavioral Risk Factor Surveillance System. Sex Transm Dis. 2018; 45: 808-812.

17. Liu Z, Chen S, Chen Q, et al. Effect of two test models and donors with different donation times on residual risk of HIV after detection. Chin J Blood Transfusion. 2019; 32: 585-587.

18. O'Brien SF, Yi QL, Fan W, et al. Current incidence and estimated residual risk of transfusiontransmitted infections in donations made to Canadian Blood Services. Transfusion. 2007; 47: 316325.

19. Feng Q, Yang Z, Pan H, et al. Evaluation of HIV prevalence rate and residual risk after blood screening for first/repeated unpaid blood donors in Qingdao. Chin J Blood Transfusion. 2012; 25: 868-869.

20. Shannon K, Crago AL, Baral SD, et al. The global response and unmet actions for HIV and sex workers. Lancet. 2018; 392: 698-710.

21. Hao Y, Cui Y, Sun X, et al. A retrospective study of HIV /AIDS situation: a ten-year implementation of “four frees and one care” policy in China. Chin J Dis Control Prev. 2014; 18: 369-374.

22. NCAIDS, NCSTD, China CDC. Update on the AIDS/STD epidemic in China and main response in control and prevention in January, 2016. Chin J AIDS STD. 2016; 22: 141.

23. Hui S, Xu Y, Wang L, et al. Characteristics of heterosexual transmission of HIV infection in 4 provinces in China. Disease Surveillance. 2011; 26: 458-462.

24. Dong MJ, Peng B, Liu ZF, et al. The prevalence of HIV among MSM in China: a large-scale systematic analysis. BMC Infect Dis. 2019; 19: 1000.

25. Wang L, Wang L, Norris JL, et al. HIV prevalence and influencing factors analysis of sentinel surveillance among men who have sex with men in China, 2003 - 2011. Chin Med J (Engl). 2012; 125: 1857-1861.

26. Gao Z, Zhang Y, Shan H, et al. A 30-year systematic review and meta-analysis of hepatitis B virus among blood donors in mainland China: revealing increase of new threats. Transfusion. 2017; 57: 1988-1997.

27. Chen X, Hu W, Huang C, et al. Further analysis of border ELISA specimens by confirmatory tests and NATs. Chin J Blood Transfus. 2015; 28: 198-199.

28. Lv M, Ling X. Analysis of the results of the initial grey zone donors by HIV1/2 ELISA. Chin J Blood Transfus. 2017; 30: 1387-1388.

29. Chen H, Chen J, Zhang S, et al. Significance of setting the scope of ELISA "gray zone" for blood test safety and blood source protection of blood donors. Chin J Blood Transfus. 2015; 28: 1140-1141.

30. GAO JB. Necessity of setting gray zone for HIV enzyme-linked immunosorbent test. Chin J AIDS STD. 2016; 22: 1009-1010. 
31. Liu J, Xiao L, Wang J, et al. Results of HIV antibody screening test and confirmatory test: a comparative analysis. Chin J Public Health. 2019; 35: 851-853.

32. Winsper C, Ganapathy R, Marwaha S, et al. A systematic review and meta-regression analysis of aggression during the first episode of psychosis. Acta Psychiatr Scand. 2013; 128: 413-421.

\section{Figures}
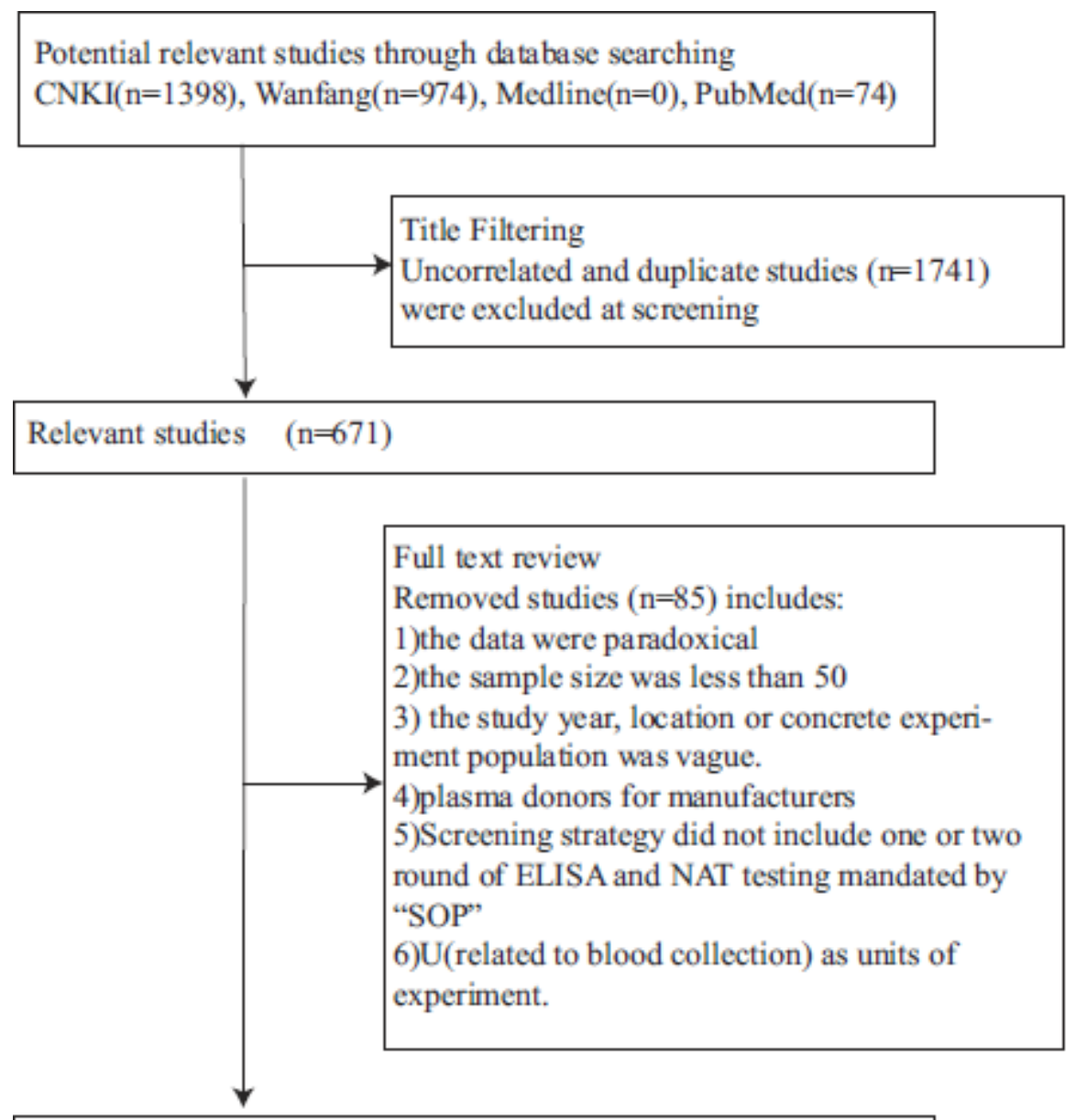

Qualified studies for analysis ( $\mathrm{n}=394)$

$\downarrow$ Subgroup Analysis

Age $(\mathrm{n}=21) \quad \operatorname{Sex}(\mathrm{n}=64) \quad$ Ethnicity $(\mathrm{n}=5) \quad$ Marriage $(\mathrm{n}=6)$

Occupation $(\mathrm{n}=22)$

Education $(\mathrm{n}=20)$

Donation frequency $(\mathrm{n}=22)$

\section{Figure 1}

PRISMA flow diagram-Synopsis of literature retrieval strategy 


\section{Potential relevant studies through database searching \\ CNKI(n=1398), Wanfang( $\mathrm{n}=974)$, Medline( $\mathrm{n}=0)$, PubMed( $\mathrm{n}=74)$}

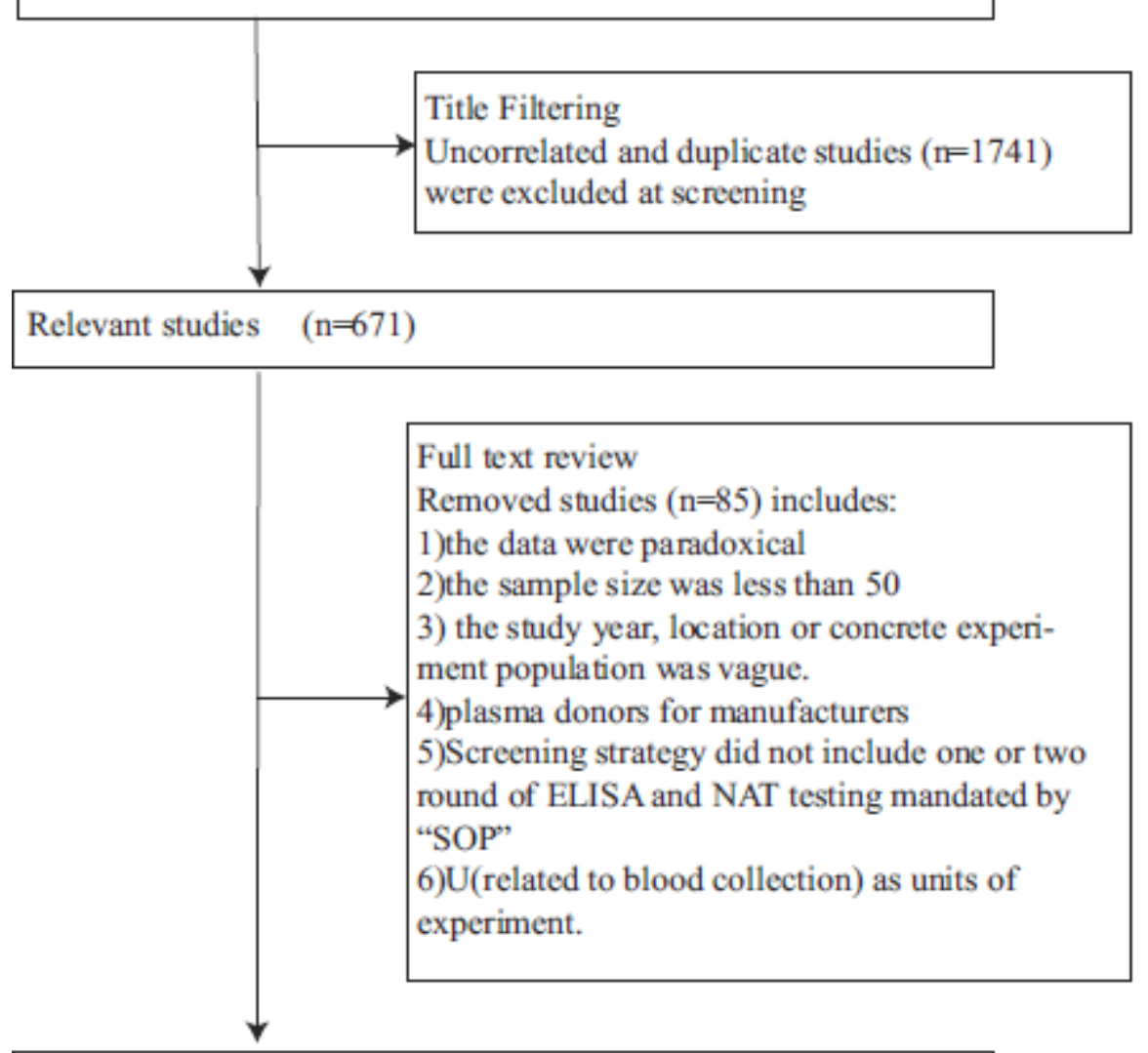

Qualified studies for analysis ( $\mathrm{n}=394$ )

\section{Subgroup Analysis}
Age $(\mathrm{n}=21)$
$\operatorname{Sex}(\mathrm{n}=64)$
Ethnicity $(\mathrm{n}=5)$
Marriage $(\mathrm{n}=6)$

Occupation $(\mathrm{n}=22)$

Education $(\mathrm{n}=20)$

Donation frequency $(\mathrm{n}=22)$

\section{Figure 2}

Provincial distribution of the pooled HIV prevalence in voluntary blood donors in mainland China. The horizontal bars of the different yellow color depth in the lower left corner indicate different ranges of rate (the base map is from the National Administration of Surveying, Mapping and Geoinformation of China [http://www.sbsm.gov.cn/] for free downloading and use). 

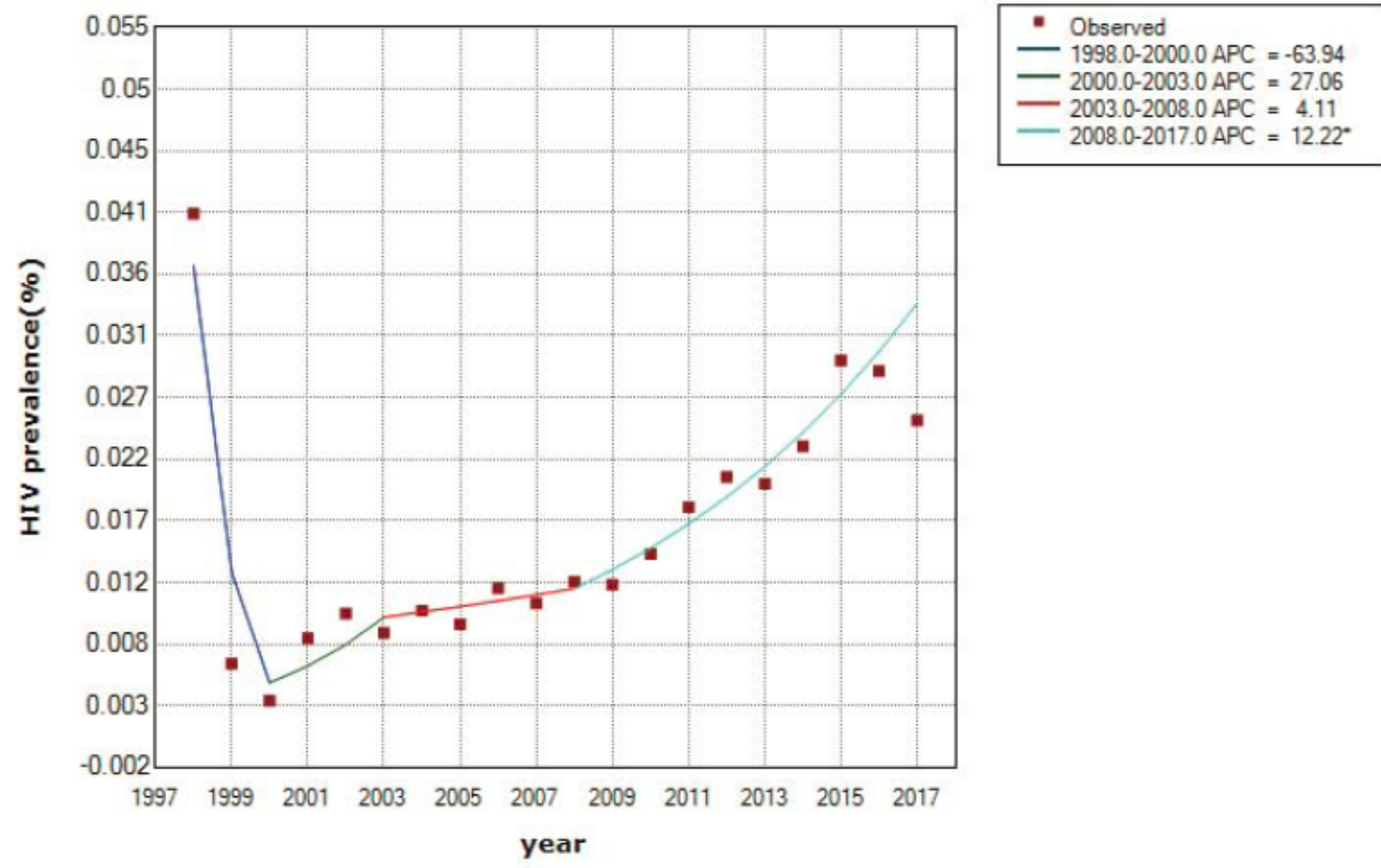

\section{Figure 3}

HIV prevalence among voluntary blood donors over the years. Percent for HIV prevalence, before 1998 to 2017. Data markers represent observed rates; lines are fitted rates based on joinpoint analysis (by Joinpoint Regression Program 4.7.0.0). The annual percentage change (APC) is significantly different from zero at $\mathrm{a}=0.05$.

\section{Supplementary Files}

This is a list of supplementary files associated with this preprint. Click to download.

- appendixlegend.docx

- appendix.docx

- OnlineSupplementalFigure1.png

- OnlineSupplementalFigure2.png 